\section{Comparación preoperatoria entre pruebas de coagulación y tromboelastografía en pacientes con cirrosis hepática sometidos a trasplante hepático}

\author{
MARIO CONCHA P., VERÓNICA MERTZ K., \\ GABRIEL MUÑOZ CASTILLO ${ }^{a}$, ALEJANDRO DELFINO Y., \\ LUIS CORTÍNEZ F., RODRIGO MONTAÑA R., \\ JUAN CRISTÓBAL PEDEMONTE T., RICARDO FUENTES H.
}

\section{Thromboelastography and classical coagulation tests in the preoperative period of liver transplantation}

Background: Compared to standard coagulation essays (SCE), such as international normalized ratio (INR), prothrombin activated partial thromboplastin time (aPTT), or platelet count, thromboelastograhy (TEG) offers precise and real-time information about hemostasis. TEG tests both platelet function and coagulation by assaying several parameters of clot formation dynamically in whole blood. Aim: To evaluate hemostasis in cirrhotic patients undergoing liver transplantation and determine the positive and negative predictive values of SCE for alterations of TEG. Material and Methods: Preoperative SCE and TEG were prospectively analyzed in 25 patients. Results were categorized as normal, laboratory alteration or clinical alteration. SCE results were compared with TEG parameters to determine positive (PPV) and negative predictive values $(N P V)$. Results: Hemostasis was abnormal and laboratory abnormalities were observed in all patients. One patient had clinical signs of excessive bleeding. SCE were abnormal in all patients and TEG was normal in nine patients. The most common alteration in TEG was hypocoagulability, in some cases associated with hypercoagulability and hyperfibrinolysis. Two patients had solely hypercoagulability. PPV of INR, aPTT, platelet count and fibrinogenemia were 0, $0,0.5$ and 0.17 respectively. NPV of the same tests were 1, 1, 0.34 and 1 respectively. Conclusions: Hypocoagulability was the most common laboratory alteration, however, clinical signs of coagulopathy were rarely present. SCE had a poor predictive value to diagnose o discard hemostatic abnormalities.

(Rev Med Chile 2018; 146: 277-281)

Key words: Blood Coagulation Disorders; Blood Coagulation Tests; Liver Cirrhosis; Liver Transplantation; Thromboelastography.

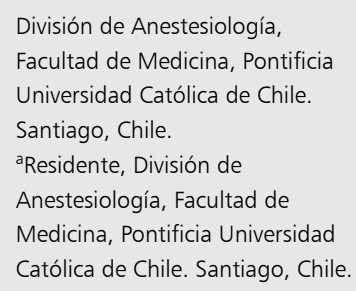

División de Anestesiología,

Facultad de Medicina, Pontificia Universidad Católica de Chile.

Santiago, Chile.

aResidente, División de

Anestesiología, Facultad de

Medicina, Pontificia Universidad

Católica de Chile. Santiago, Chile.

No hubo fuentes de apoyo

financiero.

Recibido el 27 de abril de 2017, aceptado el 23 de marzo de 2018.

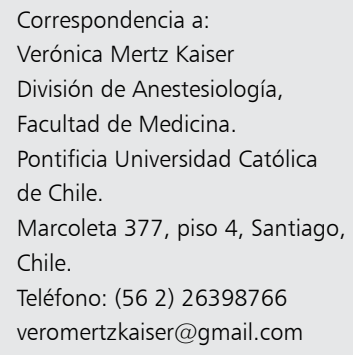

T radicionalmente, se ha asumido que los pacientes portadores de cirrosis hepática $(\mathrm{CH})$, al tener disminuida la síntesis hepática de factores de coagulación, presentan un estado de hipocoagulabilidad, con un mayor riesgo de sangrado espontáneo y en relación a procedimientos invasivos. Esto no considera que simultáneamente se ve afectada la síntesis de fac- 
tores anticoagulantes y moduladores de la acción plaquetaria y que, como resultado de esto, se genera un nuevo equilibrio hemostático, funcional, pero inestable $e^{1,2}$. Los exámenes de coagulación estándar (ECE) como el International normalized ratio (INR), la protrombinemia o el tiempo de tromboplastina parcial activada (TTPA), junto al recuento de plaquetas y los niveles plasmáticos de fibrinógeno, evalúan solo etapas o sustratos aislados de la hemostasia, sin considerar la interacción entre ellos ni con el endotelio vascular. Por esto, su capacidad para entregar información acerca del verdadero estado de la hemostasia es limitada ${ }^{3}$. La tromboelastografía (TEG) analiza el proceso de formación, estabilización y lisis del trombo ${ }^{4}$. $\mathrm{Al}$ realizarse en condiciones muy similares a las presentes en vivo, entrega una información más precisa de las características visco-elásticas de la sangre, permitiendo, además, visualizar en forma gráfica, en tiempo casi real, un trazado que refleja las etapas de generación del trombo. Por estos motivos ha sido incorporado en el diagnóstico y como guía de tratamiento de las alteraciones hemostáticas presentes en numerosas situaciones clínicas $^{4-8}$. Dentro del perfil de la TEG se han definido una serie de parámetros, como el tiempo de reacción o $\mathrm{R}$, ángulo alfa, amplitud máxima (MA) y porcentaje de lisis (LY) (Figura 1). Cada uno de estos parámetros se asocia a la disponibilidad o actividad de los diferentes elementos de la hemostasia, los cuales habitualmente se miden en forma individual con los ECE. El objetivo de este estudio fue describir el estado de la hemostasia analizado por TEG y ECE en pacientes portadores de $\mathrm{CH}$ sometidos a trasplante hepático $(\mathrm{TH}) \mathrm{y}$, a partir de ello, determinar el valor predictivo positivo (VPP) y el valor predictivo negativo (VPN) de los exámenes de coagulación habituales en relación a los parámetros del TEG relacionados con ellos.

\section{Pacientes y Método}

El estudio fue aprobado por el Comité de Ética de la Facultad de Medicina (Número 15-091 con fecha 5 de mayo de 2015). Se analizaron prospectivamente los registros clínicos preoperatorios, TEG, TTPA, INR, recuento de plaquetas y fibrinógeno, tomados al llegar a pabellón de los pacientes sometidos a trasplante hepático con diagnóstico de hepatitis fulminante o $\mathrm{CH}$ de cualquier etiología, operados entre mayo de 2015 y abril de 2016. Se excluyeron los pacientes con patología hepática tumoral y aquellos que recibieron plasma fresco congelado, plaquetas, crioprecipitado, vitamina $\mathrm{K}$ o heparina en las $24 \mathrm{~h}$ previas a la cirugía. Las muestras para estos exámenes se tomaron a la llegada del paciente a pabellón, para ser enviadas al laboratorio de urgencia para su procesamiento. El TEG utilizó caolín como activador. De acuerdo al resultado de estos exámenes, el estado de la coagulación se clasificó de la siguiente manera:

- Normal: Definida como ausencia de clínica o de alteración de ECE o TEG.

- Alteración de laboratorio: Definida como ECE o TEG alterados sin signos clínicos de sangrado.

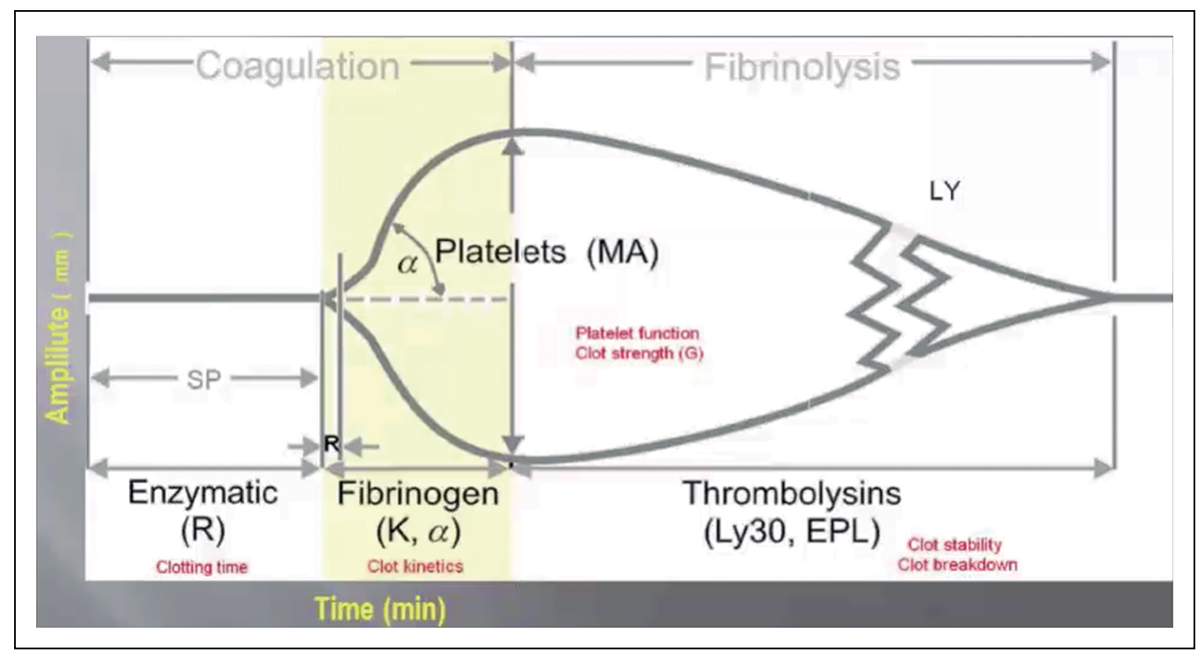

Figura 1. 
- Alteración clínica: Definida como sangrado espontáneo de piel y mucosas no atribuibles a hipertensión portal.

Se definió como alteración de exámenes a INR $>2$, TTPA $>60$ segundos, plaquetas $<50.000 / \mathrm{ml}$ y fibrinógeno $<200 \mathrm{mg} / \mathrm{ml}$.

De acuerdo al resultado de la TEG, los pacientes fueron clasificados en los siguientes grupos:

- Normal

- Hipocoagulable, definido por la presencia de alguna de la siguientes condiciones: $\mathrm{R}>10 \mathrm{~min}, \mathrm{MA}<50 \mathrm{~mm}$, o ángulo $\alpha<45^{\circ}$

- Fibrinolítico, se definió por la presencia de LY30 > 7,5\%

- Hipercoagulable, definido por MA $>73 \mathrm{~mm}$ o $\mathrm{R}<4$ min.

Los pacientes con más de una alteración se mostrarán por separado.

En relación a la normalidad o anormalidad de la TEG, se determinó el VPP y VPN de INR y TTPA para alteraciones de $\mathrm{R}$, recuento plaquetario para alteraciones de MA y de fibrinogenemia para alteraciones de ángulo alfa.

Los VPP y VPN se calcularon de la siguiente manera:

$\mathrm{VPP}=\mathrm{a} /(\mathrm{a}+\mathrm{b})$

a: verdaderos positivos para la alteración

b: falsos positivos para la alteración

VPP: proporción con que se identifica correctamente a los sujetos en una situación clínica determinada; es la razón entre verdaderos positivos y el total de positivos.

$\mathrm{VPN}=\mathrm{d} /(\mathrm{c}+\mathrm{d})$

c: falsos negativos para la alteración

d: verdaderos negativos para la alteración

VPN: proporción con la que se descarta la enfermedad en una situación clínica determinada. Es la razón entre los verdaderos negativos y el total de negativos.

\section{Resultados}

En el período estudiado se trasplantaron 27 pacientes, excluyéndose 2 de ellos por haber recibido PFC, plaquetas, o ambos, en las $24 \mathrm{~h}$ previas a la cirugía. Se estudiaron 25 pacientes, edad 55,8 $\pm 10,7$ peso $68,7 \pm 10,1 \mathrm{~kg}$, talla $163 \pm$
$7,4 \mathrm{~cm}$ y score MELD (Model For End-Stage Liver Disease) de 23,7 \pm 9 . Durante el período de estudio no ingresó ningún paciente con falla hepática fulminante. En todos los pacientes la hemostasia fue calificada como anormal, presentando todos alteración de laboratorio y solo 1 alteración clínica. Este presentaba evidencias de alteración plaquetaria y de fibrinógeno en la TEG y en los ECE. Los ECE fueron anormales en todos los pacientes. Las alteraciones encontradas en los ECE se muestran en la Tabla 1. La TEG fue normal en 9 pacientes, las alteraciones encontradas se muestran en la Tabla 2. Los valores de los parámetros de la TEG se muestran en la Tabla 3. En relación a los valores anormales definidos de la TEG, los VPP y VPN de INR, TTPA, plaquetas, y fibrinógeno fueron, respectivamente, $0,0,0,5$ y 0,17 , y los VPN 1,1 , 0,34 y 1 .

\section{Tabla 1. Alteraciones de los exámenes de coagulación estándar (ECE)}

\begin{tabular}{|lc|}
\hline INR $>2$ & 11 \\
\hline Protrombina $<40 \%$ & 14 \\
TTPA $>60 \mathrm{seg}$ & 7 \\
Plaquetas $<50.000 / \mathrm{ml}$ & 2 \\
\hline Fibrinógeno $<200 \mathrm{mg} / \mathrm{ml}$ & 23 \\
\hline
\end{tabular}

INR: international normalized ratio. TTPA: tiempo de tromboplastina parcial activada.

Tabla 2. Alteración de tromboelastografía (TEG)

\begin{tabular}{|ll|}
\hline Hipocoagulable & 9 \\
\hline Hipercoagulable & 2 \\
Hipocoagulable + hipercoagulable & 8 \\
\hline Hipocoagulable + fibrinolítico & 1 \\
\hline Hipocoagulable + hipercoagulable + fibrinolítico & 1 \\
\hline
\end{tabular}

Tabla 3. Valores de parámetros tromboelastográficos

\begin{tabular}{|lrrl|}
\hline Parámetro & \multicolumn{1}{c}{$\begin{array}{c}\text { Valores } \\
\text { encontrados }\end{array}$} & $\begin{array}{c}\text { Valores } \\
\text { normales }\end{array}$ \\
\hline R & 4,5 & 1,4 & $2-8$ min \\
\hline Ángulo & 56,5 & 14,3 & $55-78$ grados \\
MA & 44,6 & 13,4 & $51-69 \mathrm{~mm}$ \\
\hline LY & 2,2 & 7,2 & $0-8 \%$ \\
\hline
\end{tabular}

R: reaction time, MA: maximum amplitude, LY: lysis. 


\section{Discusión}

Los resultados muestran que las alteraciones de laboratorio de la hemostasia, de acuerdo con las definiciones utilizadas en este estudio, se presentan en la totalidad de los pacientes, pero se acompañan de escasa manifestación clínica. Esto es concordante con lo que se ha planteado en los pacientes con $\mathrm{CH}$, en los cuales, si bien pueden existir disminuciones de factores procoagulantes, plaquetas, proteína $\mathrm{C}$, y antitrombina, pueden también aumentar otros factores como factor VII y von Willebrand ${ }^{1,9}$. Todo lo anterior condiciona el establecimiento de un nuevo equilibrio hemostático ${ }^{2}$. Esto explica por qué estos pacientes rara vez presentan un estado de hipocoagulabilidad clínica, como generalmente se asume, pudiendo incluso desarrollar condiciones de hipercoagulabilidad, aun en pacientes con falla hepática aguda con INR $>2^{10-12}$.

En dos de los pacientes estudiados, el estado de coagulación se calificó como hipercoagulable y en 9 de ellos, esta condición se presentó junto a hiperfibrinolisis o hipocoagulabilidad (Tabla 2). El hallazgo de la coexistencia en distintos puntos del proceso hemostático de alteraciones que actúan en sentidos opuestos sobre la coagulación, es concordante con el establecimiento de un nuevo equilibrio hemostático. Este estudio confirma la presencia de otras alteraciones, como la hipercoagulabilidad y la fibrinolisis, rara vez consideradas en estos pacientes, y que pudieran estar relacionadas con el origen de morbilidad grave.

Es importante destacar que, en aquellos pacientes que tuvieron un perfil de TEG hipocoagulable, el valor de $\mathrm{R}$ fue normal. Es decir, el origen de la alteración no es la falta de factores y, por lo tanto, no es susceptible a ser corregida mediante el uso de plasma fresco congelado (PFC). Esta práctica, especialmente el uso "profiláctico" de PFC antes de la realización de procedimientos invasivos, carece de fundamento, siendo también causa de morbilidad derivada del uso de PFC o exacerbación de la hipertensión portal con un posible aumento del riesgo de sangrado ${ }^{13-16}$.

El nuevo equilibrio hemostático resulta imposible evaluar con los ECE. La discrepancia entre las pruebas estándar y la TEG ha sido reportada en cirugía resectiva hepática y durante $\mathrm{TH}^{10,17,18}$. Los ECE no fueron diseñados para evaluar el estado global de la coagulación, solo miden una parte muy inicial, pequeña y específica del proceso de hemostasia, no considerando el endotelio, los factores celulares, la presencia de factores anticoagulantes o el efecto de la fibrinolisis, careciendo así de capacidad predictora de riesgo de sangrado. Por esta razón, no debería sorprender el escaso VPP y VPN de los ECE. Desafortunadamente, en la práctica clínica habitual, y en ausencia de otros exámenes que permitan evaluar la hemostasia, se asume que los ECE sí tienen esta capacidad. Esto es motivo de acciones terapéuticas erróneas y potencialmente riesgosas. A diferencia de las ECE, y aun cuando la TEG no es considerada un gold standard para evaluar hemostasia, permite una evaluación más completa del proceso de generación y posterior lisis del trombo y de todos los elementos participantes en el proceso. De esta manera, y gracias a la estandarización de los parámetros medidos, orienta a la causa de los trastornos de la hemostasia y permite desarrollar una terapia dirigida. Para precisar más este punto se debe señalar que el valor del ángulo alfa, y más aun la MA, dependen de la cantidad y función plaquetaria y de la concentración de fibrinógeno. Para discriminar entre ambos componentes es necesario medir fibrinógeno funcional en el TEG, agregando factor tisular e inhibidor plaquetario ${ }^{19}$. En este estudio se realizó solamente TEG monoanálisis, activado con caolín, sin fibrinógeno funcional ni inhibidor plaquetario, por lo cual no es posible discriminar acerca de cuál es el responsable de la alteración observada. Esta limitación del método, ciertamente, podría modificar las conclusiones referentes a las causas de las alteraciones observadas. Es interesante destacar que en 9 pacientes la TEG fue normal y que, aun cuando el estudio no permite explicarlo, esto pudiera ser reflejo de las numerosas nuevas interacciones que se establecen entre los participantes en el proceso hemostático y que dan cuenta del nuevo equilibrio de este ${ }^{1,2}$.

Todas las consideraciones previas nos obligan a cuestionar la utilidad de los exámenes tradicionalmente utilizados para evaluar la hemostasia. La utilidad de un examen está dada por la capacidad de este de detectar una alteración cuando esta existe (VPP), y por la capacidad de descartarla cuando no lo está (VPN). Si bien este rendimiento puede verse influido por las definiciones aplicadas, la utilización de alguna de estas permite establecer un criterio objetivo de comparación. Desafortunadamente, no existe consenso para definir los 
criterios diagnósticos de una alteración, lo que dificulta estas comparaciones. Asumiendo esta limitación, la posibilidad de correlacionar algunos de los parámetros de la TEG con los ECE, además de evaluar de manera más completa el proceso de hemostasia, permite establecer algunas comparaciones respecto a los ECE. Si bien estos pueden ser herramientas útiles para evaluar la evolución de la función hepática, su limitada capacidad de estimación del proceso de hemostasia y los malos VPP y VPN los hacen muy malos evaluadores del estado de la coagulación y no dicen nada de la posible causa de una alteración cuando esta está presente.

En resumen, si bien todos los pacientes con $\mathrm{CH}$ estudiados presentaron alteraciones de laboratorio, y que la hipocoagulabilidad fue el trastorno de laboratorio más frecuente, en solo un paciente esto se acompañó de manifestaciones clínicas, siendo frecuente la presencia de otras alteraciones.

En relación a la TEG, los ECE presentaron una mala capacidad de detectar o descartar una alteración de hemostasia, por lo cual no debieran utilizarse para este objetivo.

\section{Referencias}

1. Tripodi A, Mannucci PM. The coagulopathy of chronic liver disease. N Engl J Med 2011; 365 (2): 147-56.

2. Lisman T, Caldwell SH, Burroughs AK, Northup PG, Senzolo M, Stravitz RT, et al. Hemostasis and thrombosis in patients with liver disease: the ups and downs. J Hepatol 2010; 53 (2): 362-71.

3. Tripodi A, Mannucci PM. Abnormalities of hemostasis in chronic liver disease: reappraisal of their clinical significance and need for clinical and laboratory research. J Hepatol 2007; 46 (4): 727-33.

4. Jeger V, Zimmermann H, Exadaktylos AK. Can Rapid TEG accelerate the search for coagulopathies in the patient with multiple injuries? J Trauma 2009; 66 (4): 1253-7.

5. Forfori F, Ferro B, Mancini B, Letizia R, Abramo A, Anselmino $\mathrm{M}$, et al. Role of thrombolestagrophy in monitoring perioperative coagulation status and effect of thromboprophylaxis in bariatric surgery. Obesity Surgery 2012; 22 (1): 113-8.

6. Shore-Lesserson L, Manspeizer HE, DePerio M, Francis S, Vela-Cantos F, Ergin MA. Thromboelastography-guided transfusion algorithm reduces transfusions in complex cardiac surgery. Anesth Analg 1999; 88 (2): 312-9.

7. Wang SC, Shieh JF, Chang KY, Chu YC, Liu CS, Loong
CC, et al. Thromboelastography-guided transfusion decreases intraoperative blood transfusion during orthotopic liver transplantation: randomized clinical trial. Transplant Proc 2010; 42 (7): 2590-3.

8. Carroll RC, Craft RM, Whitaker GL, Snider CC, Kirby RK, Elder RF, et al. Thrombelastography monitoring of resistance to enoxaparin anticoagulation in thrombophilic pregnancy patients. Thrombosis Research 2007; 120 (3): 367-70.

9. Tripodi A, Primignani M, Chantarangkul V, Viscardi $Y$, Dell'Era A, Fabris FM, et al. The coagulopathy of cirrhosis assessed by thromboelastometry and its correlation with conventional coagulation parameters. Thromb Res 2009; 124 (1): 132-6.

10. Krzanicki D, Sugavanam A, Mallett S. Intraoperative hypercoagulability during liver transplantation as demonstrated by thromboelastography. Liver Transpl 2013; 19 (8): 852-61.

11. Agarwal B, Wright G, Gatt A, Riddell A, Vemala V, Mallett $S$, et al. Evaluation of coagulation abnormalities in acute liver failure. J Hepatol 2012; 57 (4): 780-6.

12. Stravitz RT, Lisman T, Luketic VA, Sterling RK, Puri $\mathrm{P}$, Fuchs M, et al. Minimal effects of acute liver injury/ acute liver failure on hemostasis as assessed by thromboelastography. J Hepatol 2012; 56 (1): 129-36.

13. Weeder PD, Porte RJ, Lisman T. Hemostasis in liver disease: implications of new concepts for perioperative management. Transfus Med Rev 2014; 28 (3): 107-13.

14. Boyer JL, Chatterjee C, Iber FL, Basu AK. Effect of plasma-volume expansion on portal hypertension. N Engl J Med 1966; 275 (14): 750-5.

15. Massicotte L, Denault AY, Thibeault L, Hevesi Z, Nozza A, Roy A. Relationship between conventional coagulation tests and bleeding for 600 consecutive liver transplantations. Transplantation 2014; 98 (2): e13-5.

16. Kozek-Langenecker SA, Ahmed AB, Afshari A, Albaladejo P, Aldecoa C, Barauskas G, et al. Management of severe perioperative bleeding: guidelines from the European Society of Anaesthesiology: First update 2016. Eur J Anaesthesiol 2017; 34 (6): 332-95.

17. Bezeaud A, Denninger MH, Dondero F, Saada V, Venisse L, Huisse MG, et al. Hypercoagulability after partial liver resection. Thromb Haemost 2007; 98 (6): 1252-6.

18. Cerutti E, Stratta C, Romagnoli R, Schellino MM, Skurzak S, Rizzetto M, et al. Thromboelastogram monitoring in the perioperative period of hepatectomy for adult living liver donation. Liver Transpl 2004; 10 (2): 289-94.

19. Solomon C, Schochl H, Ranucci M, Schlimp CJ. Can the Viscoelastic Parameter alpha-Angle Distinguish Fibrinogen from Platelet Deficiency and Guide Fibrinogen Supplementation? Anesth Analg 2015; 121 (2): 289-301. 